\title{
Échanges transdisciplinaires et légitimation générique : le poème en prose et les arts plastiques
}

\section{Bertrand Bourgeois}

\section{(2) OpenEdition Journals}

Electronic version

URL: http://journals.openedition.org/transtexts/802

DOI: $10.4000 /$ transtexts.802

ISSN: 2105-2549

Publisher

Gregory B. Lee

Electronic reference

Bertrand Bourgeois, «Échanges transdisciplinaires et légitimation générique : le poème en prose et les arts plastiques », Transtext(e)s Transcultures 跨文本跨文化 [Online], 12 | 2017, Online since 09 July 2018, connection on 01 May 2019. URL : http://journals.openedition.org/transtexts/802 ; DOI :

$10.4000 /$ transtexts. 802

This text was automatically generated on 1 May 2019.

(c) Tous droits réservés 


\title{
Échanges transdisciplinaires et légitimation générique : le poème en prose et les arts plastiques
}

\author{
Bertrand Bourgeois
}

Suzanne Bernard l'a souligné dans son étude fondatrice, le poème en prose est un genre anarchique né de la révolte ${ }^{1}$ et la plupart de ses premiers praticiens ne rencontrent à l'origine qu'un succès d'estime alors que leur œuvre est sinon déconsidérée, tout au moins méconnue du grand public. Aloysius Bertrand, que Charles Baudelaire proclame l'inventeur du poème en prose, meurt dans l'anonymat, et la parution posthume de son œuvre Gaspard de la nuit en 1842 a lieu dans l'indifférence presque complète du champ littéraire. Baudelaire meurt lui aussi avant de finir Le Spleen de Paris, recueil dont on doit la parution posthume en 1869 à Théodore de Banville et Charles Asselineau. La réception contemporaine de ce recueil est pour ainsi dire inexistante, et il restera considéré par la critique, jusqu'aux années 1960, comme une œuvre mineure du poète jugée digne de peu d'intérêt. ${ }^{2}$ Les Illuminations de Rimbaud n'ont à l'origine pas plus un statut de recueil poétique volontairement constitué par son auteur, puisqu'elles paraissent sous forme de fragments successifs, grâce à Paul Verlaine, en 1886, dans quelques numéros de La Vogue, revue dont «le tirage confidentiel $»^{3}$ rappelle le lectorat restreint autant que l'impact limité qu'elle eut sur le champ littéraire de l'époque. Les aléas de la première guerre mondiale font également des Calligrammes d'Apollinaire un recueil posthume regroupant des poèmes longtemps déconsidérés par la critique : ils ont été par exemple présentés comme un "manifeste amusant [...] que l'on aurait pu croire d'un collégien ", ${ }^{4}$ ou même été réduits à de pures " tautologies ». ${ }^{5}$

2 Dans ses multiples manifestations historiques, depuis Aloysius Bertrand jusqu'aux pratiques les plus récentes, le poème en prose apparaît ainsi comme un genre hybride dont le nom antithétique résume le paradoxe qui l'anime et semble lui dénier toute possibilité d'une légitimité générique au sein de la tradition littéraire. Contrairement à la poésie versifiée qu'il délaisse délibérément, et que des critères formels bien précis permettent d'identifier, le genre marginal et marginalisé du poème en prose ne semble 
pouvoir se définir que comme ce qu'il n'est pas, à la frontière d'autres formes littéraires telles que la nouvelle, la fable ou l'anecdote, avec lesquelles il partage nombre de ses traits.

3 Délibérément mineur, refusant les codes hérités de la tradition littéraire et occupant une place résolument marginale dans le champ littéraire, le poème en prose n'a toutefois de cesse de surprendre et, dès ses balbutiements, ce sera notre hypothèse, il part en quête d'une légitimité générique du côté des arts visuels en se revendiquant comme héritier de pratiques plastiques mineures. En outre, en renonçant à la versification, il se coupe des ressorts rythmiques et musicaux propres à la poésie pour faire advenir « le miracle d'une prose poétique, musicale sans rythme et sans rime, assez souple et assez heurtée pour s'adapter aux mouvements lyriques de l'âme ", ${ }^{6}$ selon les célèbres mots de Baudelaire à Arsène Houssaye. Ce faisant, le poème en prose dénoue le lien orphique sacré qui rattachait d'abord la poésie à la musique, le vers à l'oreille, et ouvre la voie à une poésie de l'œil où les mouvements d'une plume « assez souple et assez heurtée » ne trouveraient d'équivalents que dans les coups de pinceaux de l'artiste-peintre.

4 Afin d'explorer les échanges transdisciplinaires impliqués par la revendication de légitimité plastique du poème en prose, c'est à une micro-histoire du poème en prose en mode mineur que cet article entend procéder. Il interrogera d'abord le choix effectué par quatre poètes de recourir à des dénominations qui relèvent de pratiques artistiques mineures pour désigner aussi bien leurs poèmes en prose que les recueils dans lesquels ils s'insèrent : la bambochade (Aloysius Bertrand), la caricature (Charles Baudelaire), le croquis (Baudelaire et Joris-Karl Huysmans) ou encore l'enluminure (Arthur Rimbaud). Cette micro-histoire retracera ensuite l'historique complexe de publication qui conduit Guillaume Apollinaire de l'album d'« idéogrammes lyriques » aux Calligrammes et indique que le poème en prose peut désormais se passer d'une référence générique explicite aux arts plastiques. Il s'agira ainsi de démontrer le passage d'une entreprise de légitimation générique du poème en prose à l'affirmation d'une nature proprement plastique du poème.

\section{« Un nouveau genre de prose $»^{7}$ : entre fantaisie et bambochade}

5 Les appellations dont Aloysius Bertrand entoure son Gaspard de la nuit, sont à cet égard aussi révélatrices qu'inaugurales, dans la mesure où les poètes de la fin du dix-neuvième siècle, Baudelaire le premier, l'établiront a posteriori comme le fondateur du " genre » du poème en prose. On sait en effet qu'Aloysius Bertrand n'a jamais utilisé le terme de "poème en prose " pour qualifier le «nouveau genre de prose» qu'il invente, et les termes qu'il utilise n'appartiennent pas à la tradition générique littéraire, mais plutôt aux domaines extérieurs de la musique et de la peinture. Il sous-titre le recueil «fantaisies à la manière de Rembrandt et de Callot », et affirme dans sa préface signée Gaspard de la nuit avoir réalisé, outre de telles fantaisies, « des études sur Van-Eyck, Lucas de Leyde, Albert Dürer, Peeter Neef, Breughel de Velours, Breughel d'Enfer, Van-Ostade, Gerard Dow, Salvator-Rosa, Murillo, Fusely et plusieurs autres maîtres de différentes écoles $»{ }^{8}$

6 À propos de la notion générique de "fantaisie », Luc Bonenfant a affirmé avec justesse que le recours de Bertrand à ce terme qui relève d'abord de la tradition musicale allemande lui permettait de rompre avec la tradition littéraire française, en même temps 
qu'il lui fournissait un espace de liberté créatrice à l'écart de carcans génériques et d'horizons d'attente réducteurs. ${ }^{9}$

7 À la suite de Bonenfant, Fanny Bérat-Esquier souligne «la promotion du mineur opérée par le choix de ce terme et d'une esthétique qui lui est rattachée $» \cdot{ }^{10}$ Elle dégage toutefois ce terme de la tradition musicale à laquelle Bonenfant le rattache exclusivement et insiste sur le fait qu' « une autre dimension doit être prise en compte : celle du recours à d'autres arts que l'art littéraire. Dans la fantaisie est en effet primordial le rapport à l'image ».11 Ainsi, le recours à cette désignation générique, placée sous la double égide "de Rembrandt et Callot ", aurait un autre enjeu : celui de rechercher une légitimité du côté de la peinture, voire, à travers la liste de noms propres convoqués, de se constituer une véritable paternité picturale, afin d'inscrire l'esthétique de son recueil dans la lignée des «maîtres de différentes écoles» de la peinture hollandaise, italienne, espagnole et française.

8 Le rapport au pictural, et en particulier à Rembrandt et Callot, est d'ailleurs posé, moins sur le mode de la transposition ekphrastique, puisqu'il ne s'agit pas dans le recueil de reproduire tel ou tel tableau des deux artistes, que dans la logique d'une filiation avant tout esthétique : «à la manière de » est en effet à l'origine une expression réservée à la peinture qui évoque, depuis le maniérisme de la fin du XVI ${ }^{\mathrm{e}}$ siècle, la manière, c'est-à-dire le maniement du pinceau, la facture et le style, propres à un peintre ou à une école. Bertrand justifie le choix de Rembrandt et Callot du fait qu'ils incarnent pour lui «les deux faces antithétiques $»^{12}$ de l'art :

Rembrandt est le philosophe à barbe blanche qui s'encolimaçonne en son réduit, qui absorbe sa pensée dans la méditation et dans la prière, qui ferme les yeux pour se recueillir, qui s'entretient avec des esprits de beauté, de science, de sagesse et d'amour, et qui se consume à pénétrer les mystérieux symboles de la nature. Callot, au contraire, est le lansquenet fanfaron et grivois qui se pavane sur la place, qui fait du bruit dans la taverne, qui caresse les filles de bohémiens, qui ne jure que par sa rapière et par son escopette, et qui n'a d'autre inquiétude que de cirer sa moustache. ${ }^{13}$

9 Une telle association relève avant tout d'une problématisation morale et thématique du pictural qui oppose deux conceptions de l'art et de la vie : Rembrandt, ou la philosophie et le sacré, l'art transcendantal et le domaine immatériel et symbolique des idées ; Callot, ou le plaisir physique des sens et du corps, le matérialisme d'une réalité quotidienne et triviale. Derrière ces deux postulations idéologiques, ce sont deux manières de peindre qui sont corollairement évoquées : Rembrandt et l'art subtil du clair-obscur avec sa maitrise de la lumière et des ombres; Callot et le maniement de la ligne et du dessin, l'art prosaïque de la caricature vivante et mouvante du réel..$^{14}$ Rêve picturalement impossible, l'esthétique bertrandienne chercherait donc à assimiler ces deux postulations qui correspondraient finalement à ce "nouveau genre de prose » : le poème en prose où la dualité qui oppose les termes "poème» et "prose " peut être comprise et désignée métonymiquement par les noms et les manières de Rembrandt et Callot. Rembrandt, ou le "poème ", c'est-à-dire l'idéal incarné dans une forme parfaite; Callot, ou la prose, entendue comme représentation triviale du réel.

Ce seraient donc des peintres et leurs manières qui permettraient à Aloysius Bertrand d'asseoir la légitimité générique et esthétique du poème en prose, ce que confirmerait bien l'appellation de «bambochade », ${ }^{15}$ également convoquée pour désigner les textes de son recueil. Comme le rappellent notamment Steve Murphy et Luc Bonenfant, Aloysius Bertrand avait même d'abord songé à intituler Gaspard de la nuit, "bambochades 
romantiques $»^{16}:$ «Ces trois pièces font partie d'un recueil de compositions du même genre que l'auteur se propose de publier très prochainement sous le titre de Bambochades romantiques. ${ }^{17}$ On notera d'ailleurs que l'emploi du terme "bambochade romantique " est associé aux notions de « recueil» de « compositions » et surtout de " genre ». Il serait sans doute exagéré d'affirmer que ce dernier mot ait sous la plume de Bertrand le sens précis de "genre littéraire", mais le contexte dans lequel le titre "bambochades romantiques » est évoqué par Bertrand indique bien une réflexion métapoétique.

11 Si ce titre est finalement abandonné par Bertrand, le terme de "bambochades" se retrouve dès la première ligne d' " Harlem ", texte liminaire du recueil et qui peut se lire à bien des égards comme un manifeste poétique: «Harlem, cette admirable bambochade qui résume l'école flamande, Harlem peint par Jean-Breughel, Peeter-Neef, David Téniers et Paul Rembrandt. $»^{18}$. Le mot "bambochade » est appliqué à Harlem, titre du poème, ville elle-même présentée comme la métonymie de «l'école flamande », objet du poème, et plus généralement du premier livre du Gaspard de la nuit intitulé "L'école flamande ». Les textes de ce premier livre sont ainsi à envisager comme autant de tableaux imaginaires de cette école picturale dont Aloysius Bertrand mentionne les noms les plus célèbres. "Bambochade» fait ainsi figure de terme générique qui s'applique aux différents textes du recueil. Or, il s'agit d'un terme de peinture qui évoque «Il Bamboccio ", surnom du peintre néerlandais de l'âge d'or Pieter Van Laer qui fut actif à Rome, où il reçut ce surnom signifiant «le bamboche " ou «le pantin » faisant très certainement allusion à son physique difforme, dont il affuble d'ailleurs nombre des figures grotesques qu'il représente. Le terme de «bambochade» désigne ainsi plus spécifiquement à la fois sa manière picturale et les sujets de prédilection de ce peintre : des tableaux généralement de petit format, "représentant des scènes grotesques et champêtres $»^{19}$ typiquement flamandes, telles que des scènes de beuveries dans des tavernes, des scènes de jeux de cartes, de fêtes de villages, de brigandages, et où la vie quotidienne est représentée de façon burlesque et presque caricaturale. Par extension, la «bambochade » désigne des tableaux, eaux-fortes, dessins, gravures qui, même s'ils ne sont pas d' "Il Bamboccio », exploitent cette même veine, tels ceux de Jacques Callot, David Téniers, Adriaen Van Ostade ou Adriaen Brouwer, autant de noms récurrents sous la plume d'Aloysius Bertrand.

12 En plaçant son recueil sous l'auspice de «la bambochade», ce sont donc trois caractéristiques picturales propres à ce genre qu'Aloysius Bertrand impose au poème en prose: le petit format, c'est-à-dire, en termes littéraires, un texte bref et nettement circonscrit ; la représentation du quotidien le plus prosaïque sous forme de courtes scénettes visuelles; et enfin, le grotesque et le caricatural élevés au rang du poétique, trois caractéristiques effectivement présentes dans la majorité des textes du recueil, et assurément décelables dans les poèmes en prose d'autres auteurs.

\section{Les «Petits poëmes en prose ", entre croquis de mœurs et caricature}

13 Que Baudelaire, qui se pose avec provocation dans la postérité de l'obscur Aloysius Bertrand, ait lui aussi lorgné du côté des arts visuels pour asseoir la légitimité d'un genre mineur qu'il est le premier à nommer « petits poëmes en prose » fait peu de doute : une section des Fleurs du mal s'intitule déjà " Tableaux parisiens », et le recueil qu'il hésite à appeler Le Spleen de Paris ou même Le Rôdeur parisien peut ainsi se lire comme le pendant 
de cette section qui se proposait de décrire des scènes de la vie quotidienne à Paris. ${ }^{20} \mathrm{Ce}$ n'est sans doute d'ailleurs pas un hasard si dans le poème en prose "Les projets ", la découverte d'une estampe dans une boutique de gravure par un narrateur qui déambule dans la ville constitue le point de départ de l'imagination poétique :

En passant plus tard dans une rue, il s'arrêta devant une boutique de gravures, et, trouvant dans un carton une estampe représentant un paysage tropical, il se dit [...]. 21 la mode dans la seconde moitié du XIX ${ }^{\mathrm{e}}$ siècle ${ }^{22}$ qui fascine Baudelaire et lui fournit un modèle esthétique pour ses poèmes en prose, ainsi que l'a noté Michelle Hannoosh: liberté formelle et privilège donné à l'expression individuelle, variété de tons et d'effets, pertinence pour représenter la réalité urbaine moderne seraient les trois principales caractéristiques qui feraient de «cette forme relativement mineure un modèle permettant de définir les possibilités, en fin de compte, à la fois d'une forme nouvelle et d'un art visuel et littéraire distinctement moderne ${ }^{23}$

On sait en outre que l'essai Le Peintre de la vie moderne consacré à Constantin Guys, qui parait pour la première fois en 1863 en feuilleton dans trois numéros successifs du Figaro, s'avère une façon indirecte pour Baudelaire de parler de son art, et plus précisément, de ce qu'il accomplit dans Le Spleen de Paris. Or, dans Le Peintre de la vie moderne, Baudelaire intitule la courte deuxième section « Le croquis de mœurs ", genre qu'il considère comme le plus apte à représenter la beauté mobile et «triviale» de son époque. Dans cette section, il assimile avec insistance le peintre, sinon à un poète, du moins à un écrivain :

Plus l'artiste y mettra de beauté, plus l'œuvre sera précieuse ; mais il y a dans la vie triviale, dans la métamorphose journalière des choses extérieures, un mouvement rapide qui commande à l'artiste une égale vélocité d'exécution. Les gravures à plusieurs teintes du dix-huitième siècle ont obtenu de nouveau les faveurs de la mode, comme je le disais tout à l'heure ; le pastel, l'eau-forte, l'aqua-tinte ont fourni tour à tour leurs contingents à cet immense dictionnaire de la vie moderne disséminé dans les bibliothèques, dans les cartons des amateurs et derrière les vitres des plus vulgaires boutiques. [...]. On a justement appelé les œuvres de Gavarni et de Daumier des compléments de la Comédie humaine. Balzac lui-même, j'en suis très convaincu, n'eût pas été éloigné d'adopter cette idée, laquelle est d'autant plus juste que le génie de l'artiste peintre de mœurs est un génie d'une nature mixte, c'est-à-dire où il entre une bonne partie d'esprit littéraire. [...]. Quelquefois il est poëte ; plus souvent il se rapproche du romancier ou du moraliste ; il est le peintre de la circonstance et de tout ce qu'elle suggère d'éternel. ${ }^{24}$

Favorisé par l'émergence de nouvelles techniques picturales et lithographiques (le pastel, l'eau-forte, l'aqua-tinte), le croquis de mœurs permet, selon Baudelaire, de saisir «la métamorphose journalière des choses extérieures", c'est-à-dire "la vie moderne ». L'artiste qui se livre au genre du croquis de mœurs, le « peintre de la circonstance et de tout ce qu'elle suggère d'éternel ", possède donc la capacité d'extraire la beauté du transitoire et d'en faire une œuvre d'art qui résiste au passage du temps.

Or, comme le poète en prose, il puise son inspiration « dans la vie triviale » et « derrière les vitres des plus vulgaires boutiques ", c'est-à-dire dans le prosaïsme du quotidien. Pour ce faire, il consulte l'«immense dictionnaire de la vie moderne» qui lui permet de réaliser «la description de la vie moderne, ou plutôt d'une vie moderne et plus abstraite $",{ }^{25}$ idéal revendiqué des Petits poëmes en prose. On notera en outre que les deux artistes auxquels Baudelaire fait explicitement référence dans cet extrait sont Gavarni et Daumier, c'est-à-dire deux des plus grands caricaturistes du dix-neuvième siècle: ce 
serait donc le genre de la caricature, intimement lié au croquis de mœurs, qui serait en fin de compte le genre de référence pour l'esthétique baudelairienne, et plus particulièrement pour le poème en prose.

On sait en effet l'importance que Baudelaire accorde au genre de la caricature auquel il a consacré plusieurs essais. Dans Quelques caricaturistes français, Baudelaire affirme par exemple à propos de l'œuvre de Carle Vernet: «Son œuvre est un monde, une petite Comédie humaine; car les images triviales, les croquis de la foule et de la rue, les caricatures, sont souvent le miroir le plus fidèle de la vie $»{ }^{26}$ Comme dans Le peintre de la vie moderne, le caricaturiste est une fois de plus comparé avec l'écrivain, et «les images triviales, les croquis de la foule et de la rue » qu'il livre dans ses caricatures ne sont pas sans rappeler les choses vues et les personnages croqués dans les poèmes du Spleen de Paris. On pense par exemple aux situations et aux personnages de poèmes en prose tels que " Le Mauvais vitrier ", "Les Fenêtres », «La fausse monnaie », " Le Joujou du pauvre ", ou encore "La soupe et les nuages " pour ne citer que les titres de poèmes où l'excès du trait caricatural pour rendre compte d'une prosaïque réalité témoigne à l'évidence, pour reprendre les mots de James Hiddelston, «que l'esthétique du Spleen de Paris doive quelque chose à ce genre mineur, mais éminemment moderne, de la caricature $»{ }^{27}$

\section{Le poème en prose selon Huysmans : de la bambochade au croquis parisien}

Huysmans, qui se présente lui-même comme "un inexplicable amalgame d'un parisien raffiné et d'un peintre de la Hollande $»^{28}$ et qui « a édité un volume de Croquis parisiens où, après Aloysius Bertrand et Baudelaire, il a tenté de façonner le poème en prose $»^{29}$, procéderait de fait à une étrange synthèse des modèles picturaux génériques convoqués par les deux poètes qui l'ont précédé.

Comme Bertrand, Huysmans se réfère à la bambochade, même s'il ne le revendique pas aussi directement. Le poète dijonnais est néanmoins présenté comme un prédécesseur, et la thématique des bambochades flamandes est explicitement convoquée dans la poésie de Huysmans : les "croquis de concerts et de bals de barrière ", ${ }^{30}$ les "cabarets brodés de pampre et de lierre $»^{31}$, les "paysans de Breuwer $»^{32}$ sont en effet autant de thèmes récurrents de bambochades qu'il présente comme «les principaux sujets ${ }^{33}$ de son Drageoir aux épices dans le sonnet liminaire qui a valeur de manifeste poétique, et dont une ligne biffée du manuscrit autographe assimilait même les poèmes en prose du recueil à des «tableaux fuligineux de vieux maîtres Flamands ». ${ }^{34}$ En outre, Adrien Brauwer, qui fut, on l'a dit, un peintre de bambochades, n'est pas seulement mentionné dans le sonnet liminaire, mais est l'objet même d'un des poèmes du Drageoir auquel il donne son titre. Puisqu'il s'agit « de façonner le poème en prose ", c'est-à-dire de conférer une forme à ce genre nouveau, Huysmans, à l'instar d'Aloysius Bertrand, part en quête d'une légitimité générique, non du côté des formes galvaudées de la littérature, mais du côté des genres plastiques mineurs: "vieux médaillons sculptés, / émaux, pastels pâlis, eau-forte, estampe rousse $» .{ }^{35}$

Si la bambochade n'est qu'une référence suggérée parmi ces pratiques mineures, c'est celle du croquis que Huysmans revendique explicitement à plusieurs reprises. Le terme ouvre en effet le premier vers du sonnet liminaire de son premier recueil, en même temps 
qu'il donne son titre à son second et dernier recueil de poèmes en prose, Croquis parisiens. $\mathrm{Au}$ même titre que la bambochade pour Bertrand, le croquis comme technique picturale qui sert de référence générique n'est donc pas un choix fortuit de Huysmans, et il a plusieurs implications formelles pour le poème en prose. Le Littré propose la définition suivante du croquis :

Terme d'art, surtout de peinture. Ouvrage fait à la hâte, qui n'a que les premiers traits, au-dessous encore de l'esquisse. Faire le croquis d'une figure. Esquisse d'un ensemble dont les détails ne sont pas terminés. Par extension. Il a jeté sur le papier un croquis de son poëme. ${ }^{36}$

Le caractère mineur d'une pratique "au-dessous encore de l'esquisse" ressort à l'évidence de cette définition peu élogieuse du Littré. La rapidité d'exécution et l'inachèvement sont deux traits propres à ce genre mineur selon le dictionnaire du XIX ${ }^{\mathrm{e}}$ siècle. La mise en relation avec la notion d'« esquisse " - convoquée à deux reprises mérite toutefois d'être notée, surtout quand l'on se rappelle ce que Diderot en dit à propos de Fragonard dans son Salon de 1767 : « Pourquoi une belle esquisse nous plait-elle plus qu'un beau tableau? C'est qu'il y a plus de vie et moins de formes $» .^{37}$ L'écrivaincritique d'art a bien saisi ce qui fait la force de ce genre mineur, quand il est l'œuvre d'un peintre de talent : son inachèvement formel est le garant même d'un plus grand réalisme dans l'expression de la vie en mouvement.

La définition huysmansienne du croquis se situe à l'évidence dans cet héritage diderotien, via Baudelaire. Pour Huysmans, les trois traits soulignés par le Littré - inachèvement, hâte, caractère mineur - s'avèrent en effet les véritables qualités d'un genre dont elles garantissent même la modernité, et partant, celle du poème en prose. L'héritage de la modernité baudelairienne renforce explicitement celui de la définition de l'esquisse par Diderot: le titre Croquis parisiens renvoie directement à l'expression «tableaux parisiens ", titre de la deuxième section des Fleurs du mal, en même temps qu'il évoque aussi indirectement les petits poèmes en prose de Baudelaire, et leur autre titre, Le Spleen de Paris.

Enfin, la notion de croquis fait signe vers Constantin Guys, «le peintre de la vie moderne ", dont Baudelaire a fait le symbole de sa conception esthétique du beau et qu'il a présenté comme le maître du croquis de mœurs. Et Huysmans de situer explicitement dans la lignée de Guys, un de ses amis peintres qu'il admire: Jean-Louis Forain. Il le présente en effet comme «l'un des peintres de la vie moderne les plus incisifs que je connaisse $»^{38}$ et affirme que Forain «a voulu ce que le Guys, révélé par Baudelaire, avait fait pour son époque $»{ }^{39}$ Huysmans inscrit ainsi sa modernité dans une double lignée symétrique et légitimatrice : si Forain (qui illustre d'ailleurs les Croquis parisiens de Huysmans) est le nouveau Guys, Huysmans se présente dès lors presque explicitement, et en toute modestie, comme le nouveau Baudelaire.

De la bambochade bertrandienne, en passant par le croquis de mœurs et la caricature vantés par Baudelaire, au poème en prose "croquis parisien ", il s'agit pour Huysmans d'envisager le poète en prose comme un artiste capable de saisir un réel prosaïque en mouvement dans un texte bref et dense qui ne s'encombre pas de détails inutiles. Une telle définition du poème en prose comme croquis incisif de la réalité quotidienne rejoindrait en outre la célèbre définition générique idéale du poème en prose fantasmée par le personnage de des Esseintes dans À rebours en 1884 :

De toutes les formes de la littérature, celle du poème en prose était la forme préférée de des Esseintes. Maniée par un alchimiste de génie, elle devait, suivant 
lui, renfermer, dans son petit volume, à l'état d'of meat, la puissance du roman dont elle supprimait les longueurs analytiques et les superfétations descriptives. [...]

Le roman, ainsi conçu, ainsi condensé en une page ou deux, [...].

En un mot, le poème en prose représentait, pour des Esseintes, le suc concret,

l'osmazome de la littérature, l'huile essentielle de l'art. ${ }^{40}$

En littérature, comme dans les autres arts, des Esseintes refuse les hiérarchies établies et fait du poème en prose un concentré d'art dont la brièveté dense est garante de l'efficacité narrative et esthétique: idéal littéraire par excellence qu'il substitue avec provocation au roman. Les genres picturaux mineurs du croquis, et plus généralement de l'estampe et de la gravure, fourniraient paradoxalement à Huysmans des modèles génériques novateurs qui permettraient au poème en prose de se définir comme un antiroman subvertissant ainsi les codes d'un genre devenu à la fin du dix-neuvième siècle, le genre littéraire de référence.

\section{Illuminations : Le poème en prose, entre gravure coloriée et enluminure}

C'est encore un genre mineur des arts visuels qui semble constituer l'horizon d'attente générique des Illuminations de Rimbaud, même s'il est difficile d'établir qui de Rimbaud ou de Verlaine est le principal responsable de ce choix. On sait en effet que Rimbaud n'est pour rien dans la publication de ces textes, qui paraissent pour la première fois, sous l'impulsion de Paul Verlaine, dans quelques numéros de la revue La Vogue sous le titre Les Illuminations en 1886. Dans la Notice qui les accompagne, Paul Verlaine donne la précision suivante: "Le mot Illuminations est anglais et veut dire gravures coloriées, - coloured plates : c'est même le sous-titre que M. Rimbaud avait donné à son manuscrit ». ${ }^{41}$

S'il est impossible de vérifier l'authenticité de ces propos, une phrase célèbre d'Une saison en enfer de Rimbaud semble aller dans le même sens: "J'aimais les peintures idiotes, dessus de portes, décors, toiles de saltimbanques, enseignes, enluminures populaires ${ }^{42}$.» Ces mots écrits à la première personne appartiennent d'ailleurs à la section « Alchimie du verbe » souvent lue comme une profession de foi du poète. En outre, ce goût revendiqué pour des genres picturaux mineurs fait pendant à l'expression d'un rejet des formes picturales et poétiques reconnues : «Depuis longtemps, je me vantais de posséder tous les paysages possibles et trouvais dérisoires les célébrités de la peinture et de la poésie moderne ${ }^{43}$ Formes auxquelles Rimbaud substitue notamment des pratiques graphiques mineures qu'il invite ainsi à lire comme horizon générique pour ses textes.

Quoi qu'il en soit, la notice de Verlaine dans La Vogue conditionne la réception inaugurale des Illuminations en les estampillant, par le détour de la langue étrangère, du cachet d'une technique picturale mineure à l'écart de tout modèle générique littéraire connu, celui des "gravures coloriées», et plus généralement de l'art de l'enluminure. Le Littré définit ainsi le terme « enluminures» :

Action d'enluminer ; l'art de l'enlumineur; objet enluminé. L'enluminure de cette estampe n'est pas soignée. Cela n'est pas peint, ce n'est qu'une enluminure. Nom des peintures qui ornaient autrefois les manuscrits. ${ }^{44}$

Le verbe « enluminer » est quant à lui défini comme l'action consistant à « ajouter avec le pinceau des couleurs vives sur une estampe qui lui donnent de l'éclat par rapport au trait noir ; ce qui fait comparer ces couleurs à une lumière ${ }^{45}$. » De telles définitions autorisent sans aucun doute à reconsidérer ce que Renée Hubert-Riese affirme à propos du titre et 
du sous-titre : « Painted Plates attire plutôt l'attention sur un ensemble coloré, mais stable, alors qu'illuminations' fait songer à un éclairage momentané et variable ${ }^{46}$ Le mot « illuminations » serait en effet à comprendre avant tout dans son sens anglais, synonyme de « painted plates » ou de « coloured plates ${ }^{47}$

Rimbaud, par le truchement de Verlaine, ferait de ses textes un ensemble coloré à l'aune de l'enluminure : un genre mineur de la peinture, - le ton dépréciatif du Littré est clair ("n'est pas soignée », «ce n'est que de l'enluminure ») - où ce qui importe est moins le sujet que Rimbaud semble également préférer mineur - "idiot ", " naïf», " niais ", " petit», "vieux», " populaire ${ }^{48}$ sont les adjectifs qu'il utilise dans "Alchimie du verbe » - que sa lumineuse mise en couleur. Au même titre que la bambochade pour Aloysius Bertrand, ou le croquis pour Huysmans, le modèle générique de la gravure coloriée permet à « l'homme aux semelles de vent » de faire du poème en prose un genre plastique mineur où l'accent est mis sur la production d'images poétiques éclatantes et contrastées, ainsi qu'en témoigne un poème tel que « Fleurs » :

D'un gradin d'or, - parmi les cordons de soie, les gazes grises, les velours verts et les disques de cristal qui noircissent comme du bronze au soleil, - je vois la digitale s'ouvrir sur un tapis de filigranes d'argent, d'yeux et de chevelures.

Des pièces d'or jaune semées sur l'agate, des piliers d'acajou supportant un dôme d'émeraudes, des bouquets de satin blanc et de fines verges de rubis entourent la rose d'eau.

Tels qu'un dieu aux énormes yeux bleus et aux formes de neige, la mer et le ciel attirent aux terrasses de marbre la foule des jeunes et fortes roses. ${ }^{49}$

Le poème fait en effet figure de véritable enluminure où scintillent couleurs et matières chatoyantes sur un fond d'or, si bien que les fleurs du titre sont moins décrites que suggérées sous la forme d'impressions colorées et lumineuses par l'art d'un poète-joailler qui les transforme en autant de pierres précieuses textuelles.

Michel Murat emporte ainsi notre adhésion quand il souligne l'adéquation générique qui unit le titre des Illuminations aux poèmes qui composent le recueil : "C'est une trouvaille de poète, si congruente à son objet qu'elle est capable de fonder un genre nouveau, un titre puissamment synthétique, et en même temps ambigu ou glissant $»{ }^{50} \mathrm{Il}$ semblerait cependant que ce soit avant tout dans " son rapport à l'image et au discours sur l'image " ${ }^{51}$ que ce titre ébauche les fondations d'un genre nouveau.

\section{De l'album d'idéogrammes lyriques aux Calligrammes: au-delà de la légitimité picturale}

Alors que se multiplient les avant-gardes artistiques à l'aube de la première guerre mondiale, Apollinaire ne semble dorénavant plus chercher un modèle du côté des techniques et genres plastiques mineurs pour légitimer une pratique poétique en prose qu'il veut plus avant-gardiste encore que le futurisme ou le cubisme. Le 10 août 1914, Apollinaire rédige l'achevé d'imprimer d'une plaquette intitulée «Et moi aussi je suis peintre» composée d'une série d'«idéogrammes lyriques». L'Allemagne vient de déclarer la guerre à la France le 3 août, et Apollinaire se retrouve mobilisé à Nîmes en décembre, si bien que la plaquette «Et moi aussi je suis peintre » n'est pas publiée du vivant du poète. ${ }^{52}$ Comme le résume Daniel Grojnowski : «Sa plaquette passe à la trappe des désastres de l'Histoire ${ }^{5}{ }^{53}$ Les poèmes qui composent cette plaquette sont ensuite intégrés aux Calligrammes dont ils ne constituent plus qu'une mince partie. Ils perdent 
ainsi autant leur spécificité picturale originelle que leur caractère proprement expérimental :

Pour restituer le caractère propre des idéogrammes, il faut donc revenir au moment d'exaltation où Apollinaire les conçoit et les agence en album. Désignant de ce mot une plaquette fabriquée à petit nombre, il pose un cadre. [...] les «idéogrammes lyriques et coloriés " sont antérieurs aux Calligrammes qui les absorberont par la suite. Ils s'en distinguent d'autant plus nettement que leur agencement en une suite de sept pages emprunte un beau format : $29 \mathrm{~cm}$ de hauteur pour une double page de $39 \mathrm{~cm}$ de large, un espace où le lyrisme verbal se transforme en construction visuelle. L'auteur de l'album rompt les frontières qui séparent les Belles-Lettres des Beaux-Arts. ${ }^{54}$

Si Apollinaire ne prend pas à proprement parler pour modèle une technique ou un genre plastique mineur pour nommer sa pratique poétique, comme l'avaient fait avant lui Aloysius Bertrand, Huysmans ou Rimbaud, le choix de regrouper une série de poèmes baptisés «idéogrammes lyriques » en un album intitulé «Et moi aussi je suis peintre » relève véritablement d'une entreprise poétique inventant de nouveaux genres et de nouvelles pratiques qui se veulent explicitement d'ordre visuel et graphique :

Je dis idéogramme parce que, après cette production [Lettre-Océan], il ne fait plus de doute que certaines écritures modernes tendent à entrer dans l'idéographie. [...]. On m'objectera qu'un pur idéogramme est un pur dessin et ne saurait comprendre de langage écrit. Je répondrai que, dans la Lettre-Océan, ce qui s'impose et l'emporte c'est l'aspect typographique, précisément l'image, soit le dessin. Que cette image soit composée de fragments de langage parlé, il n'importe psychologiquement, car le lien entre ces fragments n'est plus celui de la logique grammaticale, mais celui d'une logique idéographique aboutissant à un ordre de disposition spatiale tout contraire à celui de la juxtaposition discursive..$^{55}$

Le Littré définit en effet l'idéogramme comme le « nom donné aux signes qui n'expriment ni une lettre ni un son quelconque, mais une idée, abstraction faite du son par lequel cette idée est rendue dans telle ou telle langue. [...]. Dans les écritures hiéroglyphiques, signes présentant des images d'idées et de choses $" .{ }^{56}$ Ainsi, le recours à une telle pratique rattache immédiatement l'entreprise poétique à la représentation imagée, abstraite et spatiale plutôt qu'à une représentation discursive reposant sur une succession de signifiés linguistiques. Pour reprendre les mots de Grojnowski, l'idéogramme vise à « accomplir une œuvre plastique dont le lyrisme « poétique » emprunterait les voies de la typographie et de la mise en page $",{ }^{57}$ c'est-à-dire qu'il s'agit de créer une poésie qui repose d'abord sur une conception proprement picturale d'un support matériel qu'ont en commun la littérature et les arts plastiques, celui de la page.

Le néologisme calligrammes qu'Apollinaire forge ensuite pour baptiser le nouveau genre de poème qu'il a inventé confirme une telle idée. Le Trésor informatisé de la langue française définit en effet le calligramme comme un «texte écrit dont les lignes sont disposées en forme de dessins $»^{58}$ avant de rappeler l'étymologie à partir de laquelle Apollinaire a forgé le mot: "néologisme créé par G. Apollinaire par croisement de idéogramme avec calligraphie $»{ }^{59}$. En convoquant la notion de calligraphie, Apollinaire met l'accent sur la beauté plastique du texte/poème envisagé comme «belle écriture », et il insiste ainsi plus nettement encore sur la révolution poétique à laquelle il procède : un lyrisme qui relève de la disposition de symboles graphiques regroupés sur une page dans un but avant tout formel :

J'ai cherché avec mes idéogrammes à retrouver une forme qui, sans être le vers libre, ne retombait pas dans le vers classique. Mes images ont la valeur d'un vers. Ils ont une forme typographique ou lapidaire déterminée... ${ }^{60}$ 
I lon notera qu'en insistant ainsi sur la plasticité formelle du poème en prose, l'entreprise poétique d'Apollinaire, dont il n'a de cesse de souligner le caractère novateur, retrouve paradoxalement la composante essentielle de la poésie classique : le vers, mais un vers désormais converti en idéogramme.

Si le poème en prose est bien dès l'origine un genre mineur et anarchique, en marge du champ littéraire, qui nait du refus (voire de la contestation) des traditions littéraires et qui se définit par des pratiques et des formes individuelles plutôt que par des lois structurelles internes, il semble que les poètes en prose, d'Aloysius Bertrand à Rimbaud, dans leur entreprise même de dénomination et de définition de leur pratique scripturale, cherchent tous de façon plus ou moins consciente, et plus ou moins explicite selon les cas, à conférer une légitimité à ce genre dont le nom même est une contradiction.

À une époque où le lyrisme et la poésie sont en crise et à réinventer, ${ }^{61}$ c'est à l'extérieur de leur domaine qu'ils vont chercher cette légitimité, en regardant obliquement du côté des techniques et des genres mineurs des arts visuels : bambochade, croquis, gravure coloriée , ou enluminure sont autant de dénominations plastiques qui permettent aux poètes de redéfinir le lyrisme autant que d'assigner une légitimité générique et théorique à la nouveauté de leur pratique poétique, où le poème en prose est pensé comme une forme d'expression éminemment visuelle :

Le poète qui se déclare peintre peint avec des mots: cette expression est ici littéralement observée, et ce sont bien les mots qui tracent un dessin, esquissent une forme, etc. Selon un certain degré d'accommodation, il est permis de discerner des figures et, dans les relations qui s'établissent entre les motifs ainsi déterminés, se dégage quelque chose comme un tableau. Telle est bien la justification de l'ut pictura poesis : donner à voir avec des mots. ${ }^{62}$

Ces lignes d'Henri Scepi à propos des idéogrammes lyriques d'Apollinaire nous semblent pouvoir être étendues à la révolution poétique à l'œuvre depuis Aloysius Bertrand, dans la mesure où elles révèlent à la fois ce qui unit et sépare Apollinaire des poètes en prose qui l'ont précédé : comme pour eux, il s'agit pour lui de peindre avec des mots. La singularité de sa démarche consiste toutefois à proclamer la possibilité de le faire littéralement: de la bambochade ou du croquis à l'idéogramme lyrique ou au calligramme se joue le passage d'une logique de la transposition, où le titre choisi relève d'un emprunt lexical direct aux arts plastiques, à une logique de la substitution, où le titre pose le poème en nouveau genre pictural, dans des titres dont les enjeux génériques sont incontestables. La transformation de la logique titulaire transpositionnelle mise à l'œuvre par plusieurs poètes en prose du XIX siècle en logique substitutive par Apollinaire traduit une rupture épistémologique fondamentale pour la poésie moderne : le passage d'une quête de légitimité du poème en prose à l'écart des modèles génériques littéraires à l'affirmation d'une autonomie visuelle du poème-tableau qui ouvre la voie à la " poésie plastique $»^{63}$ des avant-gardes de la première moitié du $\mathrm{XX}^{\mathrm{e}}$ siècle. 


\section{NOTES}

1. Suzanne Bernard, Le poème en prose de Baudelaire jusqu'à nos jours, Paris, Nizet, 1959.

2. Jusqu'à l'étude fondatrice de Charles Mauron, Le dernier Baudelaire, Paris, Corti, 1966. Pour une étude informée de la question, voir Robert Kopp, «Le Spleen de Paris: une reconnaissance tardive », in Steve Murphy (dir.), Lectures du Spleen de Paris, Rennes, PUR, 2014, p. 23-39.

3. Jean-Marie Seillan, «La Revue de poésie, une forme éditoriale hybride », Loxias, $\mathrm{n}^{\circ} 6,2004$, URL : http://revel.unice.fr/loxias/index.html?id=77, consulté le 16 octobre 2017.

4. Pascal Pia, Apollinaire par lui-même, Paris, Seuil, 1965, p. 173.

5. Michel Foucault, Ceci n'est pas une pipe, Paris, Fata Morgana, 1973, p. 21.

6. Charles Baudelaire, "À Arsène Houssaye », Le Spleen de Paris, Paris, Gallimard, 2006, p. 104. La dédicace à Houssaye est parue pour la première fois dans La Presse, le 26 août 1862.

7. C'est l'expression employée par Aloysius Bertrand dans une lettre adressée à son ami David d'Angers et datée du 18 septembre 1837.

8. Aloysius Bertrand, Gaspard de la nuit, Paris, LGF, 2002, p. 59.

9. Luc Bonenfant, «Aloysius Bertrand et les noms du genre », University of Toronto Quarterly, vol. 71, n³, 2002, p. 715.

10. Fanny Bérat-Esquier, « Gaspard de la nuit : des fantaisies en mode mineur », in Steve Murphy (dir.), Lectures de Gaspard de la nuit, de Louis ("Aloysius ») Bertrand, Rennes, Presses Universitaires de Rennes, 2010, p. 153.

11. Ibid.

12. Bertrand, ibid., p. 59.

13. Ibid.

14. La description de Rembrandt peut d'ailleurs être lue comme une évocation du célèbre tableau du maître qui se trouve au Louvre, Le Philosophe en méditation, et où s'exprime tout son art du clair-obscur.

15. Bertrand, ibid., p. 69.

16. Steve Murphy, «Avant-propos : pour le roi ou pour les rats? Notes en marges de Gaspard de la nuit ", in Steve Murphy (dir.), Lectures de Gaspard de la nuit, de Louis ("Aloysius») Bertrand, Rennes, Presses Universitaires de Rennes, 2010, p. 15.

17. Aloysius Bertrand, Le Provincial, 12 septembre 1828, p. 212, cité par Luc Bonenfant, «Le vers détourné : Aloysius Bertrand et la réinvention de la prose ", Romantisme, n¹23, 2004, p. 45.

18. Bertrand, Gaspard de la nuit, p. 69.

19. Définition du Littré : http://www.littre.org/definition/bambochade. Consulté le 30 octobre 2017.

20. Voir Patrick Labarthe, Petits poèmes en prose. Le Spleen de Paris de Charles Baudelaire, Paris, Gallimard, 2000, p. 13-15.

21. Baudelaire, « Les projets », Le Spleen de Paris, Paris, Gallimard, 2006, p. 160.

22. Ce dont témoigne la création de la Société des aquafortistes à Paris en mai 1862. Baudelaire participe de cette mode en rédigeant deux articles consacrés à l'eau-forte : «L'eau forte est à la mode » (avril 1862) et « Peintres et aquafortistes » (septembre 1862).

23. Michele Hannoosh, «Etching and Modern Art: Baudelaire's Peintres et aquafortistes », French Studies, Vol. XLIII (1), 1989, p. 60. Ma traduction. Pour plus de détails sur les liens qui unissent l'esthétique de l'eau-forte et celle du poème en prose chez Baudelaire, voir l'article complet d'Hannoosh. 
24. Baudelaire, «Le peintre de la vie moderne » [1863], L’Art romantique, Paris, Calmann Lévy, 1885, p. 56-57.

25. Baudelaire, « À Arsène Houssaye » [1862], Le Spleen de Paris, Paris, Gallimard, 2006, p. 103-104.

26. Baudelaire, «Quelques caricaturistes français » [1854], Curiosités esthétiques VII, Paris, Michel Lévy frères, 1868, p. 389.

27. James A. Hiddleston, «Les poèmes en prose de Baudelaire et la caricature ", Romantisme, $\mathrm{n}^{\circ} 74$, 1991 , p. 57.

28. Portrait autobiographique de Huysmans, publié en 1885 dans Les Hommes d'aujourd'hui, Paris, Vanier, fascicule $n^{\circ} 23$, sous le pseudonyme d'A. Meunier, cité par Patrice Locmant dans son édition critique du Drageoir aux épices, Paris, Champion, 2003, p. 249.

29. Ibid., p. 251.

30. Huysmans, Le Drageoir aux épices, Paris, Champion, 2003, p. 69.

31. Ibid.

32. Ibid.

33. Ibid.

34. Ibid., p. 68. Il s'agit de la reproduction d'une page du manuscrit qui se trouve à la bibliothèque de l'Arsenal (Fonds Lambert, n'20, folio 8).

35. Ibid., p. 69.

36. http://www.littre.org/definition/croquis. Consulté le 30 octobre 2017.

37. Denis Diderot, «Salon de 1767 », Euvres complètes, vol. 11, Nendeln, Kraus Reprint, 1875-1877, p. 245.

38. Huysmans, «J.-L. Forain », Écrits sur l'art, Paris, GF, 2008, p. 122. Ce texte paraît pour la première fois dans L'art moderne, Paris, Charpentier, 1883.

39. Ibid., p. 268. Ce texte paraît d'abord dans Certains, Paris, Stock, 1889.

40. Huysmans, À rebours, Paris, GF, 2004, p. 227. [1884].

41. Arthur Rimbaud, Les Illuminations, notice de Paul Verlaine, Paris, Publications de la Vogue, 1886.

42. Rimbaud, Une saison en enfer, Paris, Gallimard, 1999, p. 192. [1873]. On se souvient qu'Une saison en enfer est le seul texte publié par Rimbaud lui-même.

43. Ibid.

44. http://www.littre.org/definition/enluminure consultée le 30 octobre 2017.

45. http://www.littre.org/definition/enluminer consultée le 30 octobre 2017.

46. Renée Hubert-Riese, "La Technique de la peinture dans le poème en prose ", Cahiers de l'association internationale des études françaises, $\mathrm{n}^{\circ} 18,1966$, p. 175.

47. L'hésitation entre les deux expressions est également de Verlaine qui parle de "painted plates » dans une lettre à Charles de Sivry.

48. Rimbaud, Une saison en enfer, Paris, Gallimard, p. 192.

49. Rimbaud, Illuminations, Paris, GF, 2010, p. 276-277.

50. Michel Murat, L'art de Rimbaud, Paris, José Corti, 2013, p. 206.

51. Ibid.

52. Michel Décaudin, puis Daniel Grojnowski en ont toutefois récemment proposé une belle réédition : Daniel Grojnowski, «Et moi aussi je suis peintre ». Les idéogrammes d'Apollinaire, Paris, Le temps qu'il fait, 2006.

53. Ibid., notice de Grojnowski à cette édition, non paginée.

54. Ibid.

55. Gabriel Arbouin, «Devant l'idéogramme d'Apollinaire », Les Soirées de Paris, juillet-août 1914, p. 383-385. Article reproduit dans l'édition déjà citée de Grojnowski. Selon Grojnowski, Arbouin serait un pseudonyme d'Apollinaire qui théoriserait ainsi sa propre œuvre dans un périodique d'avant-garde. Il existe toutefois une notice de la Bibliothèque Nationale de France dédiée à Gabriel Arbouin (1878-1917), homme de lettres contemporain d'Apollinaire et lui aussi mort 
pendant la première guerre mondiale qui permet de douter d'une telle affirmation. Cette notice est consultable en ligne: http://data.bnf.fr/11043797/gabriel_arbouin/ consultée le 30 octobre 2017.

56. http://www.littre.org/definition/idéogramme consulté le 30 octobre 2017.

57. Grojnowski, édition de «Et moi aussi je suis peintre». Les idéogrammes d'Apollinaire, Paris, Le temps qu'il fait, 2006, non paginée.

58. http://www.cnrtl.fr/definition/calligramme consulté le 30 octobre 2017.

59. Ibid.

60. Guillaume Apollinaire, «Lettre privée à Fagus », citée par Henri Scepi, « Apollinaire entre le texte et l'image ", Critique, vol. 727, $\mathrm{n}^{0} 12,2007$, p. 979.

61. Les travaux sont nombreux sur cette question, voir notamment Jean-Michel Maupoix, $\mathrm{Du}$ lyrisme, Paris, Corti, 2000.

62. Henri Scepi, «Apollinaire entre le texte et l'image », Critique, vol. 727, n’12, 2007, p. 984.

63. Selon l'expression forgée par Isabelle Chol pour rendre compte de la poésie de Reverdy: Isabelle Chol, Pierre Reverdy. Poésie plastique, Genève, Droz, 2006.

\section{ABSTRACTS}

Genre oxymoron qui rejette la versification et la musicalité traditionnellement associées à la poésie, le poème en prose semble immédiatement se tourner vers les arts visuels. Afin d'explorer les échanges transdisciplinaires impliqués par la revendication de légitimité plastique du poème en prose, cet article procède à une micro-histoire du poème en prose en mode mineur. Il s'agit ainsi de démontrer le passage d'une entreprise de légitimation générique du poème en prose à l'affirmation d'une nature proprement plastique du poème. L'article interroge d'abord le choix effectué par quatre poètes en prose de recourir à des dénominations qui relèvent de pratiques artistiques mineures pour désigner leurs poèmes en prose : la bambochade (Aloysius Bertrand), le croquis (Charles Baudelaire, Joris-Karl Huysmans) ou encore l'enluminure (Arthur Rimbaud). L'article s'intéresse ensuite à l'historique complexe de publication des Calligrammes d'Apollinaire qui révèle l'autonomisation d'une poésie en prose plastique à la légitimité générique désormais acquise.

Prose poetry starkly challenges the versification and musicality traditionally associated with poetry. As a result, its visual aspect has often heralded the prose poem's entry into the realm of the visual arts. This article draws a micro-history of the prose poem as a minor genre, in order to explore the transdisciplinary exchanges implied by this claim of visual generic legitimacy. After first considering the choice of four poets to name their prose poetry with clear reference to visual arts genres and practices: bambochades (Aloysius Bertrand), sketches (Charles Baudelaire, Joris-Karl Huysmans), and illuminations (Arthur Rimbaud), this article analyses the convoluted publishing history of Apollinaire's Calligrammes. These case studies demonstrate how the prose poem distances itself from generic references to the visual arts, and ultimately asserts its own visual and plastic autonomy. 


\section{AUTHOR}

\section{BERTRAND BOURGEOIS}

Bertrand Bourgeois is a Lecturer in French Studies at the University of Melbourne. He is the author of Poétique de la maison-musée (1848-1898). Du réalisme balzacien à l'œuvre d'art décadente (Paris : L'Harmattan, 2009). He is currently working on a second monograph entitled Au-delà de la peinture: le poème en prose, du texte-tableau à l'objet visuel (1842-1948). He has also written various articles on nineteenth and twentieth century French authors such as Flaubert, Gautier, Goncourt, Huysmans, Perec and Houellebecq.

Bertrand Bourgeois est maître de conférences en langue et littérature françaises à l'université de Melbourne. Il est l'auteur de Poétique de la maison-musée (1848-1898). Du réalisme balzacien à l'œuvre d'art décadente (Paris : L'Harmattan, 2009) et termine actuellement un second ouvrage intitulé $\mathrm{Au}$ delà de la peinture : le poème en prose, du texte-tableau à l'objet visuel (1842-1948). Spécialiste de Huysmans, il a également consacré plusieurs articles à des romanciers du XIX et $\mathrm{XX}^{\mathrm{e}}$ siècles tels que Flaubert, Gautier, Goncourt, Perec et Houellebecq. 